\title{
Lagrangian particle paths and ortho-normal quaternion frames
}

\author{
J D Gibbon ${ }^{1}$ and D D Holm ${ }^{1,2}$ \\ ${ }^{1}$ Department of Mathematics, Imperial College, London SW7 2AZ, UK \\ 2 Computer and Computational Science, Los Alamos National Laboratory, \\ MS D413 Los Alamos, NM 87545, USA \\ E-mail: j.d.gibbon@ic.ac.uk,d.holm@ic.ac.uk and dholm@lanl.gov
}

Received 20 February 2007, in final form 11 May 2007

Published 12 June 2007

Online at stacks.iop.org/Non/20/1745

Recommended by D Lohse

\begin{abstract}
Experimentalists now measure intense rotations of Lagrangian particles in turbulent flows by tracking their trajectories and Lagrangian-average velocity gradients at high Reynolds numbers. This paper formulates the dynamics of an orthonormal frame attached to each Lagrangian fluid particle undergoing three-axis rotations, by using quaternions in combination with Ertel's theorem for frozen-in vorticity. The method is applicable to a wide range of Lagrangian flows including the three-dimensional Euler equations and its variants such as ideal magneto-hydrodynamics. The applicability of the quaterionic frame description to Lagrangian averaged velocity gradient dynamics is also demonstrated.
\end{abstract}

Mathematics Subject Classification: 76B03, 76F02, 76M99

\section{Introduction}

\subsection{General background}

On 16 October 1843, Hamilton wrote the expression for quaternion multiplication,

$$
\mathbb{I}^{2}=\mathbb{J}^{2}=\mathbb{K}^{2}=\mathbb{I} \mathbb{I} K=-\mathrm{Id},
$$

as an algebraic rule for composing 'turns' (directed arc lengths of great circles) in orienting his telescope. This feature-that multiplication of quaternions represents composition of rotations - has made them the technical foundation of modern inertial guidance systems in the aerospace industry where tracking the paths and the orientation of satellites and aircraft is of paramount importance [1]. The graphics community also uses them to control the orientation of tumbling objects in computer animations because they avoid the difficulties incurred at the north and south poles when Euler angles are used [2]. 


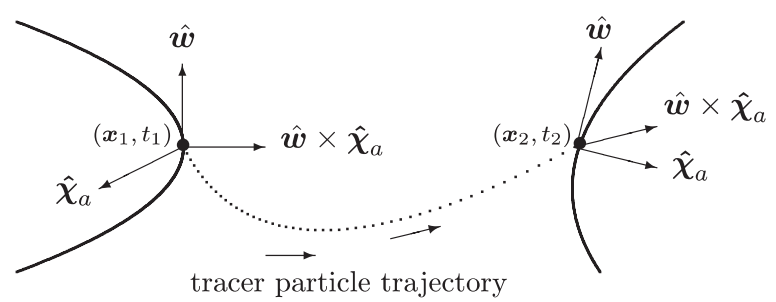

Figure 1. The dotted line represents the tracer particle $(\boldsymbol{O})$ path moving from $\left(\boldsymbol{x}_{1}, t_{1}\right)$ to $\left(\boldsymbol{x}_{2}, t_{2}\right)$. The solid curves represent characteristic curves $\boldsymbol{w}=\mathrm{d} \boldsymbol{x} / \mathrm{d} s$ ( $s$ is arc length) to which $\hat{\boldsymbol{w}}$ is a unit tangent vector. The orientation of the quaternion-frame $\left(\hat{\boldsymbol{w}}, \hat{\boldsymbol{\chi}}_{a}, \hat{\boldsymbol{w}} \times \hat{\boldsymbol{\chi}}_{a}\right)$ is shown at the two space-time points; note that this is not the Frenet-frame corresponding to the particle path but to curves $\boldsymbol{w}=\mathrm{d} \boldsymbol{x} / \mathrm{d} s$.

Given the utility of quaternions in explaining the dynamics of rotating objects in flight, one might ask whether they would also be useful in tracking the orientation and angular velocity of Lagrangian particles in fluid dynamical situations [3]. Recent experiments in turbulent flows have developed to the stage where the trajectories of tracer particles can be detected at high Reynolds numbers [4-13]; see figure 1 in [4]. Numerical differentiation of these trajectories gives information about the Lagrangian velocity and acceleration of the particles. In particular the curvature of the particle paths can be used to extract statistical information about velocity gradients from a single trajectory [13].

The usual practice in graphics problems is to consider the Frenet-frame of a trajectory which consists of the unit tangent vector, a normal and a bi-normal [2,13]. In navigational language, this represents the corkscrew-like pitch, yaw and roll of the motion. In turn, the tangent vector and normals are related to the curvature and torsion. While the Frenet-frame describes the path, it ignores the dynamics that generates the motion. Here we will discuss another ortho-normal frame associated with the motion of each Lagrangian fluid particle, designated the quaternion-frame. This may be envisioned as moving with the Lagrangian particles, but their evolution derives from the Eulerian equations of motion.

The first main contribution of this paper lies in the explicit calculation of the evolution equations of this quaternion frame. Secondly, this formulation is shown to apply to a wide range of Lagrangian problems, as the next sub-section shows. Thirdly, the evolution of the quaternion frame is related to an associated Frenet frame. Finally, it is shown how the pressure Hessian, which plays a key role in driving the quaternion frame for Euler fluid flow, can be modelled using constitutive relations.

\subsection{An appropriate class of Lagrangian flows}

Suppose $\boldsymbol{w}$ is a contravariant vector quantity attached to a particle following a flow along characteristic paths $\mathrm{d} \boldsymbol{x} / \mathrm{d} t=\boldsymbol{u}(\boldsymbol{x}, t)$ of a velocity $\boldsymbol{u}$. Let us consider the abstract Lagrangian flow equation

$$
\frac{\mathrm{D} w}{\mathrm{D} t}=\boldsymbol{a}(\boldsymbol{x}, t), \quad \frac{\mathrm{D}}{\mathrm{D} t}=\frac{\partial}{\partial t}+\boldsymbol{u} \cdot \nabla,
$$

where the material derivative has its standard definition. Examples of systems that (1.1) might represent are

1. The vector $\boldsymbol{w}$ in (1.1) could be the velocity of a tracer particle in a fluid transported by a background velocity field $\boldsymbol{u}$ with $\boldsymbol{a}$ as the particle's acceleration. 
2. $\boldsymbol{w}$ could be the vorticity $\boldsymbol{\omega}=\operatorname{curl} \boldsymbol{u}$ of the incompressible Euler fluid equations, in which case $\boldsymbol{a}=\boldsymbol{\omega} \cdot \nabla \boldsymbol{u}$ and $\operatorname{div} \boldsymbol{u}=0$. The case of the Euler equations with rotation $\Omega$ would make $\boldsymbol{w} \equiv \tilde{\boldsymbol{\omega}}=\rho^{-1}(\boldsymbol{\omega}+2 \Omega)$.

3. For the barotropic compressible Euler fluid equations (where the pressure $p=p(\rho)$ is only density dependent) then $\boldsymbol{w} \equiv \boldsymbol{\omega}_{\rho}=\rho^{-1} \boldsymbol{\omega}$, in which case $\boldsymbol{a}=\boldsymbol{\omega}_{\rho} \cdot \nabla \boldsymbol{u}$ and $\operatorname{div} \boldsymbol{u} \neq 0$.

4. The vector $\boldsymbol{w}$ could represent a small vectorial line element $\delta \ell$ transported by a background flow $\boldsymbol{u}$, in which case $\boldsymbol{a}=\boldsymbol{\delta} \boldsymbol{\ell} \cdot \nabla \boldsymbol{u}$. For example, using Moffatt's analogy between the (magnetic) $\boldsymbol{B}$-field in ideal magneto-hydrodynamics (MHD) and fluid vorticity [14], taking $\boldsymbol{\delta} \boldsymbol{\ell} \equiv \boldsymbol{B}$ in the equations for incompressible ideal MHD, then $\boldsymbol{a}=\boldsymbol{B} \cdot \nabla \boldsymbol{u}$ and $\operatorname{div} \boldsymbol{B}=0$. In a slightly generalized form it could also represent the Elsasser variables $\boldsymbol{w}^{ \pm}=\boldsymbol{u} \pm \boldsymbol{B}$ in which case $\boldsymbol{a}^{ \pm}=\boldsymbol{w}^{ \pm} \cdot \nabla \boldsymbol{u}$ with two material derivatives [14]. In each of the cases (2-4) the vectors $\boldsymbol{w}$ and $\boldsymbol{u}$ satisfy the standard Eulerian form

$$
\frac{\mathrm{D} \boldsymbol{w}}{\mathrm{D} t}=\boldsymbol{w} \cdot \nabla \boldsymbol{u}
$$

Consequently, it follows from Ertel's Theorem [15-17] that

$$
\frac{\mathrm{D}(\boldsymbol{w} \cdot \nabla \boldsymbol{\mu})}{\mathrm{D} t}=\boldsymbol{w} \cdot \nabla\left(\frac{\mathrm{D} \boldsymbol{\mu}}{\mathrm{D} t}\right)
$$

for any differentiable function $\boldsymbol{\mu}(\boldsymbol{x}, t)$. Choosing $\boldsymbol{\mu}=\boldsymbol{u}(\boldsymbol{x}, t)$ as in [16] and identifying the flow acceleration as $D \boldsymbol{u} / \mathrm{D} t=\boldsymbol{Q}(\boldsymbol{x}, t)$ yields the second derivative relation

$$
\frac{\mathrm{D}^{2} \boldsymbol{w}}{\mathrm{D} t^{2}}=\boldsymbol{w} \cdot \nabla\left(\frac{\mathrm{D} \boldsymbol{u}}{\mathrm{D} t}\right)=: \boldsymbol{w} \cdot \nabla \boldsymbol{Q}
$$

In each of the cases (2-4) above the acceleration vector $\boldsymbol{Q}$ in (1.4) is readily identifiable. Thus, in those cases we have

$$
\frac{\mathrm{D} \boldsymbol{w}}{\mathrm{D} t}=\boldsymbol{a}(\boldsymbol{x}, t) \quad \text { and } \quad \frac{\mathrm{D} \boldsymbol{a}}{\mathrm{D} t}=\boldsymbol{w} \cdot \nabla \boldsymbol{Q}=: \boldsymbol{b}(\boldsymbol{x}, t) \quad \text { along } \quad \frac{\mathrm{D} \boldsymbol{x}}{\mathrm{D} t}=\boldsymbol{u}(\boldsymbol{x}, t) .
$$

These are the kinematic rates of change of the vectors $\mathrm{D} \boldsymbol{w} / \mathrm{D} t=\boldsymbol{a}$ and $\mathrm{D} \boldsymbol{a} / \mathrm{D} t=\boldsymbol{b}$ following the characteristics of the velocity vector $\boldsymbol{u}$ along the path $\boldsymbol{x}(t)$ determined from $\mathrm{D} \boldsymbol{x} / \mathrm{D} t=\boldsymbol{u}(\boldsymbol{x}, t)$.

The plan of the paper is as follows: section 3 shows that the quartet of 3 -vectors $(\boldsymbol{u}, \boldsymbol{w}, \boldsymbol{a}, \boldsymbol{b})$ appearing in (1.5) determines the quaternion-frame and its Lagrangian dynamics: indeed, a knowledge of $\boldsymbol{b}$ is essential to determining the dynamical process. Modulo a rotation around $\boldsymbol{w}$, the quaternion-frame turns out to be the Frenet-frame attached to characteristic curves $\boldsymbol{w}=\mathrm{d} \boldsymbol{x} / \mathrm{d} s$ where $s$ is the arc length. In case (2) where $\boldsymbol{w}=\boldsymbol{\omega}$, the $\boldsymbol{w}$-curves are vortex lines and the particles are fluid parcels. As described in [16]—-see also [17] for a history-in this case Ertel's Theorem for Euler's fluid equations [15] ensures that $\boldsymbol{b}=-\mathrm{P} \boldsymbol{\omega}$ where $\mathrm{P}$ is the Hessian matrix of spatial derivatives of the pressure. In some practical situations, however, the vector $\boldsymbol{b}$ may not be known, or may not exist for every system for every triad $(\boldsymbol{u}, \boldsymbol{w}, \boldsymbol{a})$. For example no explicit relation for $\boldsymbol{b}$ is known for the Euler equations in velocity form for which $(\boldsymbol{u}, \boldsymbol{w}, \boldsymbol{a}) \equiv(\boldsymbol{u}, \boldsymbol{u},-\nabla p)$.

Section 4 elaborates three examples: the Euler equations with rotation; the barotropic compressible Euler equations; and ideal MHD in Elsasser variables. Section 5 demonstrates the applicability of the quaterionic frame description to turbulence models of Lagrangian averaged velocity gradient dynamics, in which approximate constitutive relations for the pressure Hessian and auxiliary equations for the constitutive parameters are introduced. 


\section{Quaternions and rigid body dynamics}

Rotations in rigid body mechanics has given rise to a rich and long-standing literature in which Whittaker's book is a classic example [18]. This gives explicit formulae relating the Euler angles and what are called the Cayley-Klein parameters of a rotation. In fact the use of quaternions in this area is not only much more efficient but avoids the immensely complicated inter-relations that are unavoidable when Euler angle formulae are involved $[19,20]$.

In terms of any scalar $p$ and any 3 -vector $\boldsymbol{q}$, the quaternion $\mathfrak{q}=[p, \boldsymbol{q}]$ is defined as (Gothic fonts denote quaternions)

$$
\mathfrak{q}=[p, \boldsymbol{q}]=p \mathbf{l}-\sum_{i=1}^{3} q_{i} \sigma_{i}
$$

where $\left\{\sigma_{1}, \sigma_{2}, \sigma_{3}\right\}$ are the three Pauli spin-matrices defined by

$$
\sigma_{1}=\left(\begin{array}{cc}
0 & i \\
i & 0
\end{array}\right), \quad \sigma_{2}=\left(\begin{array}{cc}
0 & 1 \\
-1 & 0
\end{array}\right), \quad \sigma_{3}=\left(\begin{array}{cc}
i & 0 \\
0 & -i
\end{array}\right),
$$

and $\mathrm{I}$ is the $2 \times 2$ unit matrix. The relations between the Pauli matrices $\sigma_{i} \sigma_{j}=-\delta_{i j} \mathrm{I}-\epsilon_{i j k} \sigma_{k}$ then give a non-commutative multiplication rule

$$
\mathfrak{q}_{1} \circledast \mathfrak{q}_{2}=\left[p_{1} p_{2}-\boldsymbol{q}_{1} \cdot \boldsymbol{q}_{2}, p_{1} \boldsymbol{q}_{2}+p_{2} \boldsymbol{q}_{1}+\boldsymbol{q}_{1} \times \boldsymbol{q}_{2}\right] .
$$

It can easily be demonstrated that quaternions are associative.

Let $\hat{\mathfrak{p}}=[p, \boldsymbol{q}]$ be a unit quaternion with inverse $\hat{\mathfrak{p}}^{*}=[p,-\boldsymbol{q}]$ : this requires $\hat{\mathfrak{p}} \circledast \hat{\mathfrak{p}}^{*}=$ $\left[p^{2}+q^{2}, 0\right]=[1,0]$ for which we need $p^{2}+q^{2}=1$. For a pure quaternion $\mathfrak{r}=[0, \boldsymbol{r}]$ there exists a transformation from $\mathfrak{r}=[0, \boldsymbol{r}] \rightarrow \mathfrak{R}=[0, \boldsymbol{R}]$

$$
\mathfrak{R}=\hat{\mathfrak{p}} \circledast \mathfrak{r} \circledast \hat{\mathfrak{p}}^{*} .
$$

This associative product can explicitly be written as

$$
\mathfrak{R}=\hat{\mathfrak{p}} \circledast \mathfrak{r} \circledast \hat{\mathfrak{p}}^{*}=\left[0,\left(p^{2}-q^{2}\right) \boldsymbol{r}+2 p(\boldsymbol{q} \times \boldsymbol{r})+2 \boldsymbol{q}(\boldsymbol{r} \cdot \boldsymbol{q})\right] .
$$

Choosing $p= \pm \cos \frac{1}{2} \theta$ and $\boldsymbol{q}= \pm \hat{\boldsymbol{n}} \sin \frac{1}{2} \theta$, where $\hat{\boldsymbol{n}}$ is the unit normal to $\boldsymbol{r}$, we find that

$$
\mathfrak{R}=\hat{\mathfrak{p}} \circledast \mathfrak{r} \circledast \hat{\mathfrak{p}}^{*}=[0, \boldsymbol{r} \cos \theta+(\hat{\boldsymbol{n}} \times \boldsymbol{r}) \sin \theta] \equiv O(\theta, \hat{\boldsymbol{n}}) \boldsymbol{r},
$$

where

$$
\hat{\mathfrak{p}}= \pm\left[\cos \frac{1}{2} \theta, \hat{\boldsymbol{n}} \sin \frac{1}{2} \theta\right] .
$$

Equation (2.6) is the famous Euler-Rodrigues formula for the rotation $O(\theta, \hat{\boldsymbol{n}})$ by an angle $\theta$ of the 3 -vector $\boldsymbol{r}$ about its normal $\hat{\boldsymbol{n}}$; the quantities $\theta, \hat{\boldsymbol{n}}$ are called the Euler parameters. The elements of the unit quaternion $\hat{\mathfrak{p}}$ are the Cayley-Klein parameters ${ }^{3}$ which are related to the Euler angles [18].

Lemma 1. The unit quaternions form a representation of the Lie group $S U(2)$.

Proof. From (2.1), the matrix representation of a unit quaternion is

$$
\mathrm{J}=p \mathrm{I}-i \boldsymbol{q} \cdot \boldsymbol{\sigma}=\left(\begin{array}{cc}
p-i q_{3} & -i q_{1}-q_{2} \\
-i q_{1}+q_{2} & p+i q_{3}
\end{array}\right)
$$

3 The Cayley-Klein parameters of the quaternion $\mathfrak{q}=[\alpha, \chi]$ in section 3 are given by

$$
\hat{\mathfrak{q}}=\left[\frac{\alpha}{\alpha^{2}+\chi^{2}}, \frac{\chi}{\alpha^{2}+\chi^{2}}\right] .
$$


where $\mathrm{J} \in S U(2)$; that is, $\mathrm{J}$ is a unitary $2 \times 2$ matrix with unit determinant. Hence, we may rewrite the map (2.4) for quaternionic conjugation equivalently in terms of the Hermitian Pauli spin matrices as

$$
\boldsymbol{R} \cdot \boldsymbol{\sigma}=\mathrm{J} \boldsymbol{r} \cdot \boldsymbol{\sigma} \mathrm{J}^{\dagger}
$$

This is the standard representation of $S O(3)$ rotations as a double covering $( \pm \mathrm{J})$ by $S U(2)$ matrices, which is now seen to be equivalent to quaternionic multiplication: for more discussion of this theorem see a modern treatise on mechanics such as [21]. The $( \pm)$ in the Cayley-Klein parameters reflects the $2: 1$ covering of the map $S U(2) \mapsto S O$ (3).

To investigate the map (2.4) when $\hat{\mathfrak{p}}$ is time-dependent, the Euler-Rodrigues formula in (2.6) can be written as

$$
\mathfrak{R}(t)=\hat{\mathfrak{p}} \circledast \mathfrak{r} \circledast \hat{\mathfrak{p}}^{*} \Rightarrow \mathfrak{r}=\hat{\mathfrak{p}}^{*} \circledast \Re(t) \circledast \hat{\mathfrak{p}} .
$$

Thus $\dot{\mathfrak{R}}$ has a time derivative given by

$$
\begin{aligned}
& \dot{\mathfrak{R}}(t)=\dot{\hat{\mathfrak{p}}} \circledast\left(\hat{\mathfrak{p}}^{*} \circledast \Re \circledast \hat{\mathfrak{p}}\right) \circledast \hat{\mathfrak{p}}^{*}+\hat{\mathfrak{p}} \circledast\left(\hat{\mathfrak{p}}^{*} \circledast \Re \circledast \hat{\mathfrak{p}}\right) \circledast \dot{\hat{\mathfrak{p}}}^{*} \\
& =\dot{\hat{\mathfrak{p}}} \circledast \hat{\mathfrak{p}}^{*} \circledast \Re+\Re \circledast \hat{\mathfrak{p}} \circledast \dot{\hat{\mathfrak{p}}}^{*} \\
& =\left(\dot{\hat{\mathfrak{p}}} \circledast \hat{\mathfrak{p}}^{*}\right) \circledast \mathfrak{R}+\mathfrak{R} \circledast\left(\dot{\hat{\mathfrak{p}}} \circledast \hat{\mathfrak{p}}^{*}\right)^{*} \\
& =\left(\dot{\hat{\mathfrak{p}}} \circledast \hat{\mathfrak{p}}^{*}\right) \circledast \Re-\left(\left(\dot{\hat{\mathfrak{p}}} \circledast \hat{\mathfrak{p}}^{*}\right) \circledast \Re\right)^{*},
\end{aligned}
$$

having used the fact on the last line that because $\mathfrak{R}$ is a pure quaternion, $\mathfrak{R}^{*}=-\mathfrak{R}$. Because $\hat{\mathfrak{p}}=[p, \boldsymbol{q}]$ is of unit length, and thus $p \dot{p}+q \dot{q}=0$, this means that $\dot{\hat{\mathfrak{p}}} \circledast \hat{\mathfrak{p}}^{*}$ is also a pure quaternion

$$
\dot{\hat{\mathfrak{p}}} \circledast \hat{\mathfrak{p}}^{*}=\left[0, \frac{1}{2} \Omega_{0}(t)\right] .
$$

The 3-vector entry in (2.12) defines the angular frequency $\Omega_{0}(t)$ as $\Omega_{0}=2(-\dot{p} \boldsymbol{q}+\dot{\boldsymbol{q}} p-\dot{\boldsymbol{q}} \times \boldsymbol{q})$ thereby giving the well-known formula for the rotation of a rigid body

$$
\dot{\boldsymbol{R}}=\boldsymbol{\Omega}_{0} \times \boldsymbol{R} .
$$

For a Lagrangian particle, the equivalent of $\Omega_{0}$ is the Darboux vector $\mathcal{D}_{a}$ in theorem 1 of section 3 .

\section{Lagrangian evolution equations and the quaternion picture}

\subsection{An ortho-normal frame and particle trajectories}

Having set the scene in section 2 by describing some of the essential properties of quaternions, it is now time to apply them to the Lagrangian relation (1.1) between the two vectors $\boldsymbol{w}$ and $\boldsymbol{a}$ which we shall repeat here

$$
\frac{\mathrm{D} w}{\mathrm{D} t}=\boldsymbol{a} \text {. }
$$

Through the multiplication rule in (2.3) quaternions appear in the decomposition of the 3vector $\boldsymbol{a}$ into parts parallel and perpendicular to another vector, which we choose to be $\boldsymbol{w}$. This decomposition is expressed as

$$
\boldsymbol{a}=\alpha_{a} \boldsymbol{w}+\chi_{a} \times \boldsymbol{w}=\left[\alpha_{a}, \chi_{a}\right] \circledast[0, \boldsymbol{w}],
$$

where the scalar $\alpha_{a}$ and 3-vector $\chi_{a}$ are defined as

$$
\alpha_{a}=w^{-1}(\hat{\boldsymbol{w}} \cdot \boldsymbol{a}), \quad \chi_{a}=w^{-1}(\hat{\boldsymbol{w}} \times \boldsymbol{a}) .
$$


Equation (3.2) thus shows that the quaternionic product is summoned in a natural manner. It is now easily seen that $\alpha_{a}$ is the growth rate of the scalar magnitude $(w=|\boldsymbol{w}|)$ which obeys

$$
\frac{\mathrm{D} w}{\mathrm{D} t}=\alpha_{a} w
$$

while $\chi_{a}$, the swing rate of the unit tangent vector $\hat{\boldsymbol{w}}=\boldsymbol{w} w^{-1}$, satisfies

$$
\frac{\mathrm{D} \hat{\boldsymbol{w}}}{\mathrm{D} t}=\chi_{a} \times \hat{\boldsymbol{w}} \text {. }
$$

Now define the two quaternions

$$
\mathfrak{q}_{a}=\left[\alpha_{a}, \chi_{a}\right], \quad \mathfrak{w}=[0, \boldsymbol{w}],
$$

where $\mathfrak{w}$ is a pure quaternion. Then (3.1) can automatically be re-written equivalently in the quaternion form

$$
\frac{\mathrm{D} \mathfrak{w}}{\mathrm{D} t}=\mathfrak{q}_{a} \circledast \mathfrak{w} .
$$

Moreover, if $\boldsymbol{a}$ is differentiable in the Lagrangian sense (see (1.5))

$$
\frac{\mathrm{D} \boldsymbol{a}}{\mathrm{D} t}=\boldsymbol{b}
$$

then, exactly as for $\mathfrak{q}_{a}$, a quaternion $\mathfrak{q}_{b}$ can be defined which is based on the variables

$$
\alpha_{b}=w^{-1}(\hat{\boldsymbol{w}} \cdot \boldsymbol{b}), \quad \chi_{b}=w^{-1}(\hat{\boldsymbol{w}} \times \boldsymbol{b}),
$$

where

$$
\mathfrak{q}_{b}=\left[\alpha_{b}, \chi_{b}\right]
$$

It is now clear that there exists a similar decomposition for $\boldsymbol{b}$ as that for $\boldsymbol{a}$ as in (3.2)

$$
\frac{\mathrm{D}^{2} \mathfrak{w}}{\mathrm{D} t^{2}}=[0, \boldsymbol{b}]=\left[0, \alpha_{b} \boldsymbol{w}+\chi_{b} \times \boldsymbol{w}\right]=\mathfrak{q}_{b} \circledast \mathfrak{w} .
$$

Using the associativity property, compatibility of (3.11) and (3.7) implies that $(w=|\boldsymbol{w}| \neq 0)$

$$
\left(\frac{\mathrm{D} \mathfrak{q}_{a}}{\mathrm{D} t}+\mathfrak{q}_{a} \circledast \mathfrak{q}_{a}-\mathfrak{q}_{b}\right) \circledast \mathfrak{w}=0,
$$

which establishes a Riccati relation between $\mathfrak{q}_{a}$ and $\mathfrak{q}_{b}$

$$
\frac{\mathrm{D} \mathfrak{q}_{a}}{\mathrm{D} t}+\mathfrak{q}_{a} \circledast \mathfrak{q}_{a}=\mathfrak{q}_{b},
$$

whose components yield

$$
\frac{\mathrm{D}}{\mathrm{D} t}\left[\alpha_{a}, \chi_{a}\right]+\left[\alpha_{a}^{2}-\chi_{a}^{2}, 2 \alpha_{a} \chi_{a}\right]=\left[\alpha_{b}, \chi_{b}\right]
$$

where $\chi_{a}=\left|\chi_{a}\right|$. From (3.13), or equivalently (3.14), there follows the first main result of the paper:

Theorem 1. The ortho-normal quaternion-frame $\left(\hat{\boldsymbol{w}}, \hat{\chi}_{\mathrm{a}}, \hat{\boldsymbol{w}} \times \hat{\chi}_{\mathrm{a}}\right) \in S O(3)$ has Lagrangian time derivatives expressed as

$$
\begin{aligned}
& \frac{\mathrm{D} \hat{\boldsymbol{w}}}{\mathrm{D} t}=\mathcal{D}_{a} \times \hat{\boldsymbol{w}}, \\
& \frac{\mathrm{D}\left(\hat{\boldsymbol{w}} \times \hat{\boldsymbol{\chi}}_{a}\right)}{\mathrm{D} t}=\mathcal{D}_{a} \times\left(\hat{\boldsymbol{w}} \times \hat{\boldsymbol{\chi}}_{a}\right), \\
& \frac{\mathrm{D} \hat{\chi}_{a}}{\mathrm{D} t}=\mathcal{D}_{a} \times \hat{\boldsymbol{\chi}}_{a},
\end{aligned}
$$

where the Darboux angular velocity vector $\mathcal{D}_{a}$ is defined as

$$
\mathcal{D}_{a}=\chi_{a}+\frac{c_{b}}{\chi_{a}} \hat{\boldsymbol{w}}, \quad c_{\mathrm{b}}=\hat{\boldsymbol{w}} \cdot\left(\hat{\chi}_{a} \times \chi_{b}\right)
$$


Remark. The Darboux vector $\mathcal{D}_{a}$ sits in a two-dimensional plane and is driven by the vector $\boldsymbol{b}$ which sits in $c_{b}$ in (3.18). The analogy with rigid body rotation expressed in (2.13) is clear.

Proof. To find an expression for the Lagrangian time derivatives of the components of the frame $\left(\hat{\boldsymbol{w}}, \hat{\boldsymbol{\chi}}_{a}, \hat{\boldsymbol{w}} \times \hat{\boldsymbol{\chi}}_{a}\right)$ requires the derivative of $\hat{\boldsymbol{\chi}}_{a}$. To find this it is necessary to use the fact that the 3-vector $\boldsymbol{b}$ can be expressed in this ortho-normal frame as the linear combination

$$
w^{-1} \boldsymbol{b}=\alpha_{b} \hat{\boldsymbol{w}}+c_{b} \hat{\chi}_{a}+d_{b}\left(\hat{\boldsymbol{w}} \times \hat{\chi}_{a}\right) .
$$

where $c_{b}$ is defined in (3.18) and $d_{b}=-\left(\hat{\chi}_{a} \cdot \chi_{b}\right)$. The 3-vector product $\chi_{b}=w^{-1}(\hat{\boldsymbol{w}} \times \boldsymbol{b})$ yields

$$
\chi_{b}=c_{b}\left(\hat{\boldsymbol{w}} \times \hat{\chi}_{a}\right)-d_{b} \hat{\chi}_{a} .
$$

When split into components, equation (3.14) becomes

$$
\frac{\mathrm{D} \alpha_{a}}{\mathrm{D} t}=\chi_{a}^{2}-\alpha_{a}^{2}+\alpha_{b}
$$

and

$$
\frac{\mathrm{D} \chi_{a}}{\mathrm{D} t}=-2 \alpha_{a} \chi_{a}+\chi_{b}
$$

From the latter it is easily seen that

$$
\frac{\mathrm{D} \chi_{a}}{\mathrm{D} t}=-2 \alpha_{a} \chi_{a}-d_{b}
$$

from which it follows

$$
\frac{\mathrm{D} \hat{\chi}_{a}}{\mathrm{D} t}=c_{b} \chi_{a}^{-1}\left(\hat{\boldsymbol{w}} \times \hat{\chi}_{a}\right), \quad \frac{\mathrm{D}\left(\hat{\boldsymbol{w}} \times \hat{\chi}_{a}\right)}{\mathrm{D} t}=\chi_{a} \hat{\boldsymbol{w}}-c_{b} \chi_{a}^{-1} \hat{\chi}_{a},
$$

which gives equations (3.15)-(3.18).

Theorem 1 is the main result of the paper and is the equivalent for a Lagrangian particle undergoing fluid motion of the well-known formula (2.13) for a rigid body undergoing rotation about its centre of mass.

\subsection{The evolution of the $\boldsymbol{b}$-field in equation (3.8)}

The Lagrangian rate of change of acceleration $\mathrm{D} \boldsymbol{a} / \mathrm{D} t=\boldsymbol{b}$ is important for tracking passive tracer particles. However, the vector $\boldsymbol{b}$ cannot be calculated directly from Ertel's theorem in (1.5). As shown below, the Lagrangian evolution of $\mathfrak{q}_{b}$ appearing in the quaternionic Riccati relation (3.13) may be described without approximation in terms of three arbitrary scalars.

Theorem 2. The Lagrangian time derivative of the quaternion $\mathfrak{q}_{b}$ in the Riccati relation (3.13) can be expressed as

$$
\begin{aligned}
\frac{\mathrm{D} \mathfrak{q}_{b}}{\mathrm{D} t} & =\mathfrak{q}_{a} \circledast \mathfrak{q}_{b}+\mathfrak{P}_{a, b}, \\
\mathfrak{P}_{a, b} & =\lambda_{1} \mathfrak{q}_{b}+\lambda_{2} \mathfrak{q}_{a}+\lambda_{3} \mathrm{Id},
\end{aligned}
$$

where $\lambda_{1}(\boldsymbol{x}, t), \lambda_{2}(\boldsymbol{x}, t), \lambda_{3}(\boldsymbol{x}, t)$ are arbitrary scalar functions and $\mathrm{Id}=[1,0]$ is the identity for the quaternions. 
Remark. Without further constraints $\lambda_{1}(x, t), \quad \lambda_{2}(x, t)$ and $\lambda_{3}(x, t)$ would be arbitrary.

Proof. To establish (3.25), we differentiate the orthogonality relation $\chi_{b} \cdot \hat{\boldsymbol{w}}=0$ and use the Lagrangian derivative of $\hat{\boldsymbol{w}}$

$$
\frac{\mathrm{D} \chi_{b}}{\mathrm{D} t}=\chi_{a} \times \chi_{b}+s_{0}, \quad \text { where } \quad s_{0}=\mu \chi_{a}+\lambda \chi_{b}
$$

$\boldsymbol{s}_{0}$ lies in the plane perpendicular to $\hat{\boldsymbol{w}}$ in which $\chi_{a}$ and $\chi_{b}$ also lie and $\mu=\mu(\boldsymbol{x}, t)$ and $\lambda=\lambda(\boldsymbol{x}, t)$ are arbitrary scalars. Explicitly differentiating $\chi_{b}=w^{-1}(\hat{\boldsymbol{w}} \times \boldsymbol{b})$ gives

$w^{-1} \hat{\boldsymbol{w}}\left(\chi_{a} \cdot \boldsymbol{b}\right)+s_{0}=-\alpha_{a} \chi_{b}-\alpha_{b} \chi_{a}+w^{-1} \hat{\boldsymbol{w}}\left(\chi_{a} \cdot \boldsymbol{b}\right)+w^{-1}\left(\hat{\boldsymbol{w}} \times \frac{\mathrm{D} \boldsymbol{b}}{\mathrm{D} t}\right)$,

which can easily be manipulated into

$$
\hat{\boldsymbol{w}} \times\left\{\frac{\mathrm{D} \boldsymbol{b}}{\mathrm{D} t}-\alpha_{b} \boldsymbol{a}-\alpha_{a} \boldsymbol{b}\right\}=w \boldsymbol{s}_{0} .
$$

This means that

$$
\frac{\mathrm{D} \boldsymbol{b}}{\mathrm{D} t}=\alpha_{b} \boldsymbol{a}+\alpha_{a} \boldsymbol{b}+\boldsymbol{s}_{0} \times \boldsymbol{w}+\varepsilon \boldsymbol{w},
$$

where $\varepsilon=\varepsilon(\boldsymbol{x}, t)$ is a third unknown scalar in addition to $\mu$ and $\lambda$ in (3.27). Thus the Lagrangian derivative of $\alpha_{b}=w^{-1}(\hat{\boldsymbol{w}} \cdot \boldsymbol{b})$ is

$$
\frac{\mathrm{D} \alpha_{b}}{\mathrm{D} t}=\alpha_{a} \alpha_{b}+\chi_{a} \cdot \chi_{b}+\varepsilon
$$

Lagrangian differential relations have now been found for $\chi_{b}$ and $\alpha_{b}$, but at the price of introducing the triplet of unknown coefficients $\mu, \lambda$, and $\varepsilon$ which are re-defined as

$\lambda=\alpha_{a}+\lambda_{1}, \quad \mu=\alpha_{b}+\lambda_{2}, \quad \varepsilon=-2 \chi_{a} \cdot \chi_{b}+\lambda_{2} \alpha_{a}+\lambda_{1} \alpha_{b}+\lambda_{3}$.

The new triplet has been subsumed into the tetrad defined in (3.26). Then (3.27) and (3.31) can again be written in the quaternion form (3.25).

In section 5 we shall discuss a approach for determining $\mathfrak{q}_{b}$ by introducing an approximate constitutive relation for the pressure Hessian $\mathrm{P}$ and auxiliary equations for the constitutive parameters.

\subsection{Frame dynamics and the Frenet equations}

Modulo a rotation around the unit tangent vector $\boldsymbol{w}$, with $\hat{\chi}_{a}$ as the unit bi-normal $\hat{\boldsymbol{b}}$ and $\hat{\boldsymbol{w}} \times \hat{\chi}_{a}$ as the unit principal normal $\hat{\boldsymbol{n}}$, the matrix $F$ can be formed (figure 2)

$$
\mathrm{F}=\left(\hat{\boldsymbol{w}}^{T},\left(\hat{\boldsymbol{w}} \times \hat{\chi}_{a}\right)^{T}, \hat{\chi}_{a}^{T}\right),
$$

and (3.15)-(3.17) can be re-written as

$$
\frac{\mathrm{DF}}{\mathrm{D} t}=\mathrm{AF}, \quad \mathrm{A}=\left(\begin{array}{ccc}
0 & -\chi_{a} & 0 \\
\chi & 0 & -c_{b} \chi_{a}^{-1} \\
0 & c_{b} \chi_{a}^{-1} & 0
\end{array}\right) .
$$

For a space curve parameterized by arc-length $s$, then the Frenet equations relating $\mathrm{dF} / \mathrm{d} s$ to the curvature $\kappa$ and the torsion $\tau$ of the curve $\boldsymbol{w}=\mathrm{d} \boldsymbol{x} / \mathrm{d} s$ are

$$
\frac{\mathrm{dF}}{\mathrm{d} s}=\mathrm{NF} \quad \text { where } \quad \mathrm{N}=\left(\begin{array}{ccc}
0 & \kappa & 0 \\
-\kappa & 0 & \tau \\
0 & -\tau & 0
\end{array}\right) \text {. }
$$




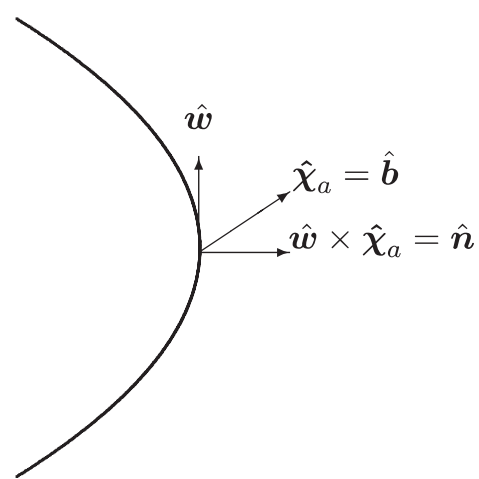

Figure 2. The ortho-normal frame $\left(\hat{\boldsymbol{w}}, \hat{\boldsymbol{w}} \times \hat{\chi}_{a}, \hat{\chi}_{a}\right)$ as the Frenet-frame to characteristic curves $\boldsymbol{w}=\mathrm{d} \boldsymbol{x} / \mathrm{d} s$.

It is now possible to relate the $t$ and $s$ derivatives of $\mathrm{F}$ given in (3.34) and (3.35). At any time $t$ the integral curves of the vorticity vector field define a space-curve through each point $\boldsymbol{x}$. The arc-length derivative $\mathrm{d} / \mathrm{d} s$ is defined by

$$
\frac{\mathrm{d}}{\mathrm{d} s}=\hat{\boldsymbol{w}} \cdot \nabla .
$$

The evolution of the curvature $\kappa$ and torsion $\tau$ of a vortex line may be obtained from Ertel's theorem in (1.3), expressed as the commutation of operators

$$
\left[\frac{\mathrm{d}}{\mathrm{d} s}, \frac{\mathrm{D}}{\mathrm{D} t}\right]=\alpha_{a} \frac{\mathrm{d}}{\mathrm{d} s}
$$

Applying this to $\mathrm{F}$ and using the relations (3.34) and (3.35) establishes the following theorem.

Theorem 3. The matrices $\mathrm{N}$ and $\mathrm{A}$ satisfy the Lax equation that relates the evolution of the curvature $\kappa$ and the torsion $\tau$ to $\alpha_{a}, \chi_{a}$ and $c_{b}$ defined in equations (3.2) and (3.18)

$$
\frac{\mathrm{DN}}{\mathrm{D} t}-\alpha_{a} \mathrm{~N}=\frac{\mathrm{dA}}{\mathrm{d} s}+[\mathrm{A}, \mathrm{N}]
$$

Thus, if $\chi_{a}=0$ the curvature $\kappa$ is stationary.

\section{Three further examples}

The quaternionic formulation can be applied to other situations, such as the stretching of fluid line-elements, incompressible and compressible motion of Euler fluids and ideal MHD [22].

(i) The Euler fluid equations for incompressible flow in a rotating frame. The velocity form of Euler's equations for an incompressible fluid flow in a frame rotating at frequency $\Omega$ is

$$
\frac{\mathrm{D} \boldsymbol{u}}{\mathrm{D} t}=\underbrace{(\boldsymbol{u} \times 2 \boldsymbol{\Omega})}_{\text {Coriolis }}-\nabla p, \quad \text { with } \quad \operatorname{div} \boldsymbol{u}=0 .
$$

Taking the curl yields $(\boldsymbol{\omega}=\operatorname{curl} \boldsymbol{u})$

$$
\frac{\mathrm{D} \tilde{\boldsymbol{\omega}}}{\mathrm{D} t}=\tilde{\boldsymbol{\omega}} \cdot \nabla \boldsymbol{u}, \quad \text { with } \quad \tilde{\boldsymbol{\omega}}=\rho^{-1}(\boldsymbol{\omega}+2 \Omega) .
$$


The triad of vectors $(\boldsymbol{u}, \boldsymbol{w}, \boldsymbol{a})$ in this case represents $(\boldsymbol{u}, \tilde{\boldsymbol{\omega}}, \tilde{\boldsymbol{\omega}} \cdot \nabla \boldsymbol{u})$ with $\boldsymbol{\omega}=\operatorname{curl} \boldsymbol{u}$ and $\operatorname{div} \boldsymbol{u}=0$. Then Ertel's theorem becomes

$$
\frac{\mathrm{D}}{\mathrm{D} t}(\tilde{\boldsymbol{\omega}} \cdot \nabla \boldsymbol{\mu})=\tilde{\boldsymbol{\omega}} \cdot \nabla\left(\frac{\mathrm{D} \boldsymbol{\mu}}{\mathrm{D} t}\right) .
$$

Upon taking $\boldsymbol{\mu}=\boldsymbol{u}$ in Ertel's theorem as in [16] and using the motion equation gives a relation in the moving frame

$$
\begin{aligned}
\frac{\mathrm{D}}{\mathrm{D} t}(\tilde{\boldsymbol{\omega}} \cdot \nabla \boldsymbol{u}) & =\tilde{\boldsymbol{\omega}} \cdot \nabla(\boldsymbol{u} \times 2 \boldsymbol{\Omega}-\nabla p) \\
& =-\mathrm{P} \tilde{\boldsymbol{\omega}}+\tilde{\boldsymbol{\omega}} \cdot \nabla(\boldsymbol{u} \times 2 \boldsymbol{\Omega}),
\end{aligned}
$$

where $P$ is the Hessian matrix of the pressure defined by

$$
\mathrm{P}=\frac{\partial^{2} p}{\partial x_{i} \partial x_{j}} .
$$

Thus (4.3) and (4.4) identify $\boldsymbol{a}$ and $\boldsymbol{b}$ as $\boldsymbol{a}=\tilde{\boldsymbol{\omega}} \cdot \nabla \boldsymbol{u}$ and $\boldsymbol{b}=-\mathrm{P} \tilde{\boldsymbol{\omega}}+\tilde{\boldsymbol{\omega}} \nabla(\boldsymbol{u} \times 2 \boldsymbol{\Omega})$; the particles are now fluid packets and not passive tracer particles. The divergence-free constraint $\operatorname{div} \boldsymbol{u}=0$ implies that

$$
-\Delta p=u_{i, j} u_{j, i}-\operatorname{div}(\boldsymbol{u} \times 2 \Omega)=\operatorname{tr} \boldsymbol{S}^{2}-\frac{1}{2} \omega^{2}-\operatorname{div}(\boldsymbol{u} \times 2 \Omega) .
$$

Equation (4.6) places an implicit condition upon the relation between the strain matrix $S$ and the pressure Hessian $P$ in addition to the Riccati equation (3.12). This situation has been discussed at greater length in [17] in the absence of rotation.

(ii) Euler's equations for a barotropic compressible fluid. The pressure of a barotropic compressible fluid is a function of its mass density $\rho$, so it satisfies $\nabla \rho \times \nabla p=0$. The velocity form of Euler's equations for incompressible fluid motion in a frame rotating at frequency $\Omega$ is

$$
\begin{aligned}
& \frac{\mathrm{D} \boldsymbol{u}}{\mathrm{D} t}=-\frac{1}{\rho} \nabla p(\rho)=:-\nabla h(\rho), \\
& \frac{\mathrm{D} \rho}{\mathrm{D} t}+\rho \operatorname{div} \boldsymbol{u}=0 .
\end{aligned}
$$

Taking the curl yields

$$
\frac{\mathrm{D} \boldsymbol{\omega}_{\rho}}{\mathrm{D} t}=\boldsymbol{\omega}_{\rho} \cdot \nabla \boldsymbol{u}, \quad \text { with } \boldsymbol{\omega}_{\rho}=\boldsymbol{\omega} / \rho \quad \text { and } \boldsymbol{\omega}=\operatorname{curl} \boldsymbol{u} .
$$

Then Ertel's theorem takes the same form as above, and the second Lagrangian time derivative yields the Ohkitani relation for a barotropic compressible fluid,

$$
\frac{\mathrm{D}^{2} \boldsymbol{\omega}_{\rho}}{\mathrm{D} t^{2}}=\frac{\mathrm{D}}{\mathrm{D} t}\left(\boldsymbol{\omega}_{\rho} \cdot \nabla \boldsymbol{u}\right)=-\boldsymbol{\omega}_{\rho} \cdot \nabla(\nabla h(\rho)) \equiv \boldsymbol{b},
$$

in terms of the Hessian of its specific enthaply, $h(\rho)$. This has the same form as for incompressible fluids, except the acceleration term $\boldsymbol{b}=-\boldsymbol{\omega}_{\rho} \cdot \nabla(\nabla h(\rho))$ has its own dynamical equation. Thus, the methods in $[17,23]$ also apply for barotropic fluids. For isentropic compressible fluids, the situation is more complicated.

(iii) The equations of incompressible ideal MHD. These are

$$
\begin{aligned}
& \frac{\mathrm{D} \boldsymbol{u}}{\mathrm{D} t}=\boldsymbol{B} \cdot \nabla \boldsymbol{B}-\nabla p, \\
& \frac{\mathrm{D} \boldsymbol{B}}{\mathrm{D} t}=\boldsymbol{B} \cdot \nabla \boldsymbol{u}
\end{aligned}
$$


together with $\operatorname{div} \boldsymbol{u}=0$ and $\operatorname{div} \boldsymbol{B}=0$. The pressure $p$ in (4.11) is $p=p_{\mathrm{f}}+\frac{1}{2} B^{2}$ where $p_{\mathrm{f}}$ is the fluid pressure. Elsasser variables are defined by the combination.

$$
\boldsymbol{v}^{ \pm}=\boldsymbol{u} \pm \boldsymbol{B} .
$$

The existence of two velocities $\boldsymbol{v}^{ \pm}$means that there are two material derivatives

$$
\frac{\mathrm{D}^{ \pm}}{\mathrm{D} t}=\frac{\partial}{\partial t}+\boldsymbol{v}^{ \pm} \cdot \nabla
$$

In terms of these, (4.11) and (4.12) can be rewritten as

$$
\frac{\mathrm{D}^{ \pm} \boldsymbol{v}^{\mp}}{\mathrm{D} t}=-\nabla p
$$

with the magnetic field $\boldsymbol{B}$ satisfying $\left(\operatorname{div} \boldsymbol{v}^{ \pm}=0\right)$

$$
\frac{\mathrm{D}^{ \pm} \boldsymbol{B}}{\mathrm{D} t}=\boldsymbol{B} \cdot \nabla \boldsymbol{v}^{ \pm}
$$

Thus we have a pair of triads $\left(\boldsymbol{v}^{ \pm}, \boldsymbol{B}, \boldsymbol{a}^{ \pm}\right)$with $\boldsymbol{a}^{ \pm}=\boldsymbol{B} \cdot \nabla \boldsymbol{v}^{ \pm}$, based on Moffatt's identification of the $\boldsymbol{B}$-field as the important stretching element [14]. From [17,23] we also have

$$
\frac{\mathrm{D}^{ \pm} \boldsymbol{a}^{\mp}}{\mathrm{D} t}=-\mathrm{P} \boldsymbol{B}
$$

where $\boldsymbol{b}^{ \pm}=-\mathrm{P} \boldsymbol{B}$. With two quartets $\left(\boldsymbol{v}^{ \pm}, \boldsymbol{B}, \boldsymbol{a}^{ \pm}, \boldsymbol{b}\right)$, the results of Section 2 follow, with two Lagrangian derivatives and two Riccati equations

$$
\frac{\mathrm{D}^{\mp} \mathfrak{q}_{a}^{ \pm}}{\mathrm{D} t}+\mathfrak{q}_{a}^{ \pm} \circledast \mathfrak{q}_{a}^{\mp}=\mathfrak{q}_{b} .
$$

In consequence, MHD-quaternion-frame dynamics needs to be interpreted in terms of two sets of ortho-normal frames $\left(\hat{\boldsymbol{B}}, \hat{\chi}^{ \pm}, \hat{\boldsymbol{B}} \times \hat{\chi}^{ \pm}\right)$acted on by their opposite Lagrangian time derivatives.

$$
\begin{aligned}
& \frac{\mathrm{D}^{\mp} \hat{\boldsymbol{B}}}{\mathrm{D} t}=\mathcal{D}^{\mp} \times \hat{\boldsymbol{B}}, \\
& \frac{\mathrm{D}^{\mp}}{\mathrm{D} t}\left(\hat{\boldsymbol{B}} \times \hat{\chi}^{ \pm}\right)=\mathcal{D}^{\mp} \times\left(\hat{\boldsymbol{B}} \times \hat{\chi}^{ \pm}\right), \\
& \frac{\mathrm{D}^{\mp} \hat{\chi}^{ \pm}}{\mathrm{D} t}=\mathcal{D}^{\mp} \times \hat{\chi}^{ \pm},
\end{aligned}
$$

where the pair of Elsasser Darboux vectors $\mathcal{D}^{\mp}$ are defined as

$$
\mathcal{D}^{\mp}=\chi^{\mp}-\frac{c_{B}^{\mp}}{\chi^{\mp}} \hat{\boldsymbol{B}}, \quad c_{B}^{\mp}=\hat{\boldsymbol{B}} \cdot\left[\hat{\chi}^{ \pm} \times\left(\chi_{p B}+\alpha^{ \pm} \chi^{\mp}\right)\right] .
$$

\section{Approximate constitutive relations for the pressure Hessian}

In this section, we discuss an approach for determining $\mathfrak{q}_{\mathrm{b}}$ by introducing an approximate constitutive relation for the pressure Hessian $P$ in the Euler equations and auxiliary equations for these constitutive parameters. Recall Euler's familiar equations for an incompressible fluid flow with velocity $\boldsymbol{u}$, written as

$$
\frac{\mathrm{D} \boldsymbol{u}}{\mathrm{D} t}=-\nabla p, \quad \text { with } \operatorname{div} \boldsymbol{u}=0
$$


Taking the gradient yields the matrix Riccati equation

$$
\frac{\mathrm{DM}}{\mathrm{D} t}+\mathrm{P}+\mathrm{M}^{2}=0
$$

Here the velocity gradient tensor $\mathrm{M}=\nabla \boldsymbol{u}$ has Cartesian components $M_{i j}=\partial u_{j} / \partial x^{i}=$ $u_{j, i}$ and the (symmetric) pressure Hessian $P=\nabla \nabla p$ has Cartesian components $\mathrm{P}_{i j}=$ $\partial^{2} p / \partial x^{i} \partial x^{j}$.

Because of the incompressibility condition $\operatorname{div} \boldsymbol{u}=0$, the trace of the velocity gradient tensor $\operatorname{tr} \mathrm{M}$ vanishes, thereby requiring $\operatorname{tr} \mathrm{P}=-\operatorname{tr}\left(\mathrm{M}^{2}\right)$, which is a Poisson equation for the pressure. For laminar flow in a bounded domain, the Poisson equation determines both the pressure in the exact Euler equations and its Hessian appearing in the velocity gradient equations (5.2).

However, in turbulent flows, modern diagnostics for both numerical simulations and fluid measurements make extensive use of average values of the velocity gradients moving in a coarse-grained volume element following the mean flow [24]. Lagrangian averaging occurs at constant fluid particle label. By its definition, Lagrangian averaging commutes with the material derivative, but with not the spatial gradient. In contrast, Eulerian averaging does the opposite. With its two Eulerian spatial gradients, the Lagrangian averaged Hessian is a challenging object to compute. Several attempts have been made to model the Lagrangian averaged pressure Hessian in (5.2) by introducing a constitutive closure for it. This idea goes back to Léorat [25], Vieillefosse [26] and Cantwell [27] who assumed that the Eulerian pressure Hessian $\mathrm{P}$ is isotropic: see also [28,29]. This assumption results in the restricted Euler equations (5.1) and (5.2) with

$$
P=-\frac{\operatorname{ld}}{3} \operatorname{tr}\left(M^{2}\right)
$$

where $\operatorname{ld}_{a b}=\delta_{a b}$ and $\operatorname{tr}(\mathrm{Id})=3$ in three dimensions, so that taking the trace satisfies the relation required for incompressibility [31]. Conversely, one may assume that the Lagrangian pressure Hessian is isotropic. The latter assumption underlies the mean flow features of the tetrad model of Chertkov et al [32]; see Chevillard and Meneveau [33] for a recent review of this approach and results on its use in turbulence diagnostics.

A more general model that encompasses the mean flow features of both the restricted Euler equations and the tetrad model emerges from the transformation properties of the pressure Hessian under the Lagrangian flow map. The pressure Hessian transforms from Eulerian to Lagrangian coordinates by the flow map $\phi_{t}: X \rightarrow x(t)$ in which $x(t)$ denotes the present position of a certain fluid particle, at time $t$, that started initially at position $X=x(0)$ at time $t=0$. This flow map preserves volume. Consequently, it is invertible and its Jacobian, the deformation gradient tensor, $\mathrm{D}_{A}^{i}(X, t)=\partial x^{i} / \partial X^{A}$ has unit determinant $\operatorname{det}(\mathrm{D})=1$. If it were frozen into the flow, the pressure Hessian would transform under the flow map as

$$
\frac{\partial^{2} p(t)}{\partial x^{i} \partial x^{j}} d x^{i}(t) \otimes d x^{j}(t)=\phi_{t} \circ\left(\frac{\partial^{2} p(0)}{\partial X^{A} \partial X^{B}} d X^{A} \otimes d X^{B}\right) .
$$

This is the way that a Riemannian metric $G$ transforms under a time-dependent change of spatial coordinates. Namely, the transformation of a Riemannian metric gives the evolving quantity $\mathrm{G}(t)$ in terms of the reference metric $\mathrm{G}(0)$ and the change of basis governed by the evolution of Jacobian matrix $\left(\mathrm{D}^{-1}\right)_{i}^{A}(t)=\partial X^{A} / \partial x^{i}$ of the inverse flow map in tensor index notation as

$$
\mathrm{G}_{i j}(t)=\mathrm{G}_{A B}(0)\left(\mathrm{D}^{-1}\right)_{i}^{A}(t)\left(\mathrm{D}^{-1}\right)_{j}^{B}(t),
$$


which relates the components of these tensors in the Eulerian and Lagrangian descriptions respectively. In short, a Riemannian metric in the Lagrangian reference configuration transforms under the flow map $\phi_{t}$ as

$$
\mathrm{G}(t)=\mathrm{D}^{-1}(t) \mathrm{G}(0) \mathrm{D}^{-1}(t),
$$

where the material time derivative of $\mathrm{D}^{-1}(t) \mathrm{d} x(t)=\mathrm{d} x(0)$ determines the evolution of $\mathrm{D}^{-1}(t) .{ }^{4}$ For the pressure Hessian to transform as a Riemannian metric and also to satisfy the Poisson equation for its trace, it must take the following algebraic form

$$
P=-\frac{G}{\operatorname{tr} G} \operatorname{tr}\left(M^{2}\right)
$$

Setting $\mathrm{G}(t)=$ Id recovers the restricted Euler equations of $[26,27]$, while setting $\mathrm{G}(0)=\mathrm{Id}$ reformulates the mean flow part of the tetrad model [32].

In fact, the Poisson equation for pressure is satisfied for any choice of the nonsingular symmetric matrix $\mathrm{G}=\mathrm{G}^{T}$ in equation (5.7) for the Hessian. Moreover, it may even be satisfied by choosing a linear combination of symmetric matrices in the form

$$
\mathrm{P}=-\left[\sum_{\beta=1}^{N} c_{\beta} \frac{\mathrm{G}_{\beta}}{\operatorname{tr} \mathrm{G}_{\beta}}\right] \operatorname{tr}\left(\mathrm{M}^{2}\right), \quad \text { with } \sum_{\beta=1}^{N} c_{\beta}=1,
$$

so long as an evolutionary flow law is provided for each of the symmetric tensors $\mathrm{G}_{\beta}=\mathrm{G}_{\beta}^{T}$ with $\beta=1, \ldots, N$. Any choice of these flow laws would also determine the evolution of the driving term $\mathfrak{q}_{\mathrm{b}}$ in the Riccati equation (3.13).

\section{Conclusions}

The review of rigid body rotations in section 2 shows that, if handled properly, quaternions have a computational advantage over Euler angle formulations. For example, the Euler-Rodrigues formula (2.6) arises from a simple multiplication of quaternions. When this approach is applied to Lagrangian evolution equations, as in section 3, it demonstrates that quaternions are a natural and efficient way of calculating the orientation and angular velocity of Lagrangian particles in motion through the introduction the concept of ortho-normal quaternion-frames travelling with each particle. For any problem in this class, knowledge of the quartet of 3 -vectors $(\boldsymbol{u}, \boldsymbol{w}, \boldsymbol{a}, \boldsymbol{b})$ is sufficient for the application of theorem 1, which is the paper's main result. The complexity of the various versions of the $3 D$ Euler equations comes through the ortho-normal dynamics via the pressure field which is itself coupled back through $\Delta p=-u_{i, j} u_{j, i}$. This has been discussed more fully in [36,37]. Adaptations of these ideas when more physics is added to the Euler equations has been discussed in section section 4. For the 3D Euler equations the pressure affects the coefficient $c_{b}$ in the Darboux vector $\mathcal{D}_{a b}$ of theorem 1 through the pressure Hessian P. The pressure Hessian also figures prominently in Lagrangian averaged models of velocity gradient dynamics in turbulence. These models employ approximate constitutive relations for the pressure Hessian. The potential applicability of the present quaternionic frame description in these Lagrangian averaged turbulence models was demonstrated in section 5.

Finally, an equivalent formulation for the compressible Euler equations may give a clue to the nature of the incompressible limit $[38,39]$.

\footnotetext{
4 The metric $\mathrm{G}(t)$ is called the Finger tensor in nonlinear elasticity. For its history and a modern application of the Finger tensor, see [30,34,35].
} 


\section{Acknowledgments}

The authors thank Trevor Stuart, Christos Vassilicos and Arkady Tsinober of Imperial College London. The work of DDH was partially supported by a Royal Society Wolfson award and by the US Department of Energy Office of Science ASCR program in Applied Mathematical Research.

\section{References}

[1] Kuipers J B 1999 Quaternions and Rotation Sequences: a Primer with Applications to Orbits, Aerospace, and Virtual Reality (Princeton, NJ: Princeton University Press)

[2] Hanson A J 2006 Visualizing Quaternions (London: Morgan Kaufmann Elsevier)

[3] Falkovich G, Gawedzki K and Vergassola M 2001 Particles and fields in fluid turbulence Rev. Mod. Phys. 73 913-75

[4] La Porta A, Voth G A, Crawford A, Alexander J and Bodenschatz E 2001 Fluid particle accelerations in fully developed turbulence Nature 409 1017-19

[5] Mordant N and Pinton J F 2001 Measurement of Lagrangian velocity in fully developed turbulence Phys. Rev. Lett. 87214501

[6] Voth G A, La Porta A, Crawford A, Bodenschatz E and Alexander J 2002 Measurement of particle accelerations in fully developed turbulence J. Fluid Mech. 469 121-60

[7] Mordant N, Crawford A M and Bodenschatz E 2004 Three-dimensional structure of the Lagrangian acceleration in turbulent flows Phys. Rev. Lett. 93214501

[8] Mordant N, Leveque E and Pinton J F 2004 Experimental and numerical study of the Lagrangian dynamics of high Reynolds turbulence New J. Phys. 6116

[9] Mordant N, Metz P, Pinton J F and Michel O 2005 Acoustical technique for Lagrangian velocity measurement Rev. Sci. Instrum. 76025105

[10] Lüthi B A, Tsinober A and Kinzelbach W 2005 Lagrangian measurement of vorticity dynamics in turbulent flow J. Fluid Mech. $\mathbf{5 2 8} 87118$

[11] Biferale L, Boffetta G, Celani A, Lanotte A and Toschi F 2005 Particle trapping in three-dimensional fully developed turbulence Phys. Fluids 17021701

[12] Reynolds A M, Mordant, Crawford A M and Bodenschatz E 2005 On the distribution of Lagrangian accelerations in turbulent flows New J. Phys. 758

[13] Braun W, De Lillo F and Eckhardt B 2006 Geometry of particle paths in turbulent flows J. Turbul. at press

[14] Moffatt H K 1978 Magnetic Field Generation in Electrically Conducting Fluids (Cambridge: Cambridge University Press)

[15] Ertel H 1942 Ein neuer Hydrodynamischer Wirbelsatz Met. Z. 59 271-81

[16] Ohkitani K 1993 Eigenvalue problems in three-dimensional Euler flows Phys. Fluids A 5 2570-2

[17] Gibbon J D, Holm D D, Kerr R M and Roulstone I 2006 Quaternions and particle dynamics in Euler fluid flow Nonlinearity 19 1969-83

[18] Whittaker E T 1944 A Treatise on the Analytical Dynamics of Particles and Rigid Bodies (New York: Dover)

[19] Klein F 2004 The Mathematical Theory of the Top: Lectures Delivered on the Occasion of the Sesquicentennial Celebration of Princeton University 2nd edn (New York: Dover Phoenix)

[20] Tait P G 1890 An Elementary Treatise on Quaternions 3rd edn (Cambridge: Cambridge University Press)

[21] Marsden J E and Ratiu T S 2003 Introduction to Mechanics and Symmetry (Texts in Applied Mathematics, vol 75 1994) (2nd printing of 2nd edn) (Berlin: Springer)

[22] Majda A J and Bertozzi A 2001 Vorticity and incompressible flow Cambridge Texts in Applied Mathematics (no 27) (Cambridge: Cambridge University Press)

[23] Gibbon J D 2002 A quaternionic structure in the three-dimensional Euler and ideal magneto-hydrodynamics equation Physica D 166 17-28

[24] Zeff B W, Lanterman D D, McAllister R, Roy R, Kostelich E and Lathrop D D 2003 Measuring intense rotation \& dissipation in turbulent flows Nature 421 146-9

[25] Léorat J 1975 Thèse de Doctorat Université Paris-VII

[26] Vieillefosse P 1994 Internal motion of a small element of fluid in an inviscid flow Physica A 125 150-62

[27] Cantwell B J 1992 Exact solution of a restricted Euler equation for the velocity gradient tensor Phys. Fluids 4 782-93

[28] Ooi A, Martin J, Soria J and Chong M S 1999 A study of the evolution and characteristics of the invariants of the velocity-gradient tensor in isotropic turbulence J. Fluid Mech. 381 141-74 
[29] Martin J, Ooi A, Chong A M and Soria J 1998 Dynamics of the velocity gradient tensor invariants in isotropic turbulence Phys. Fluids 10 2336-46

[30] Liu H and Tadmor E 2003 Critical thresholds and conditional stability for Euler equations and related models Hyperbolic Problems: Theory, Numerics, Applications Proc. 9th Int. Conf. on Hyperbolic Problems (Pasadena, CA, March 2002) ed T Hou and E Tadmor (Berlin: Springer) pp 227-40

[31] Girimaji S and Speziale C 1995 A modified restricted Euler equation for turbulent flows with mean velocity gradients Phys. Fluids 7 1438-46

[32] Chertkov M, Pumir A and Shraiman B I 1999 Lagrangian tetrad dynamics and phenomenology of turbulence Phys. Fluids 112394

[33] Chevillard L and Meneveau C 2006 Lagrangian dynamics and statistical geometric structure of turbulence Phys. Rev. Lett. 97174501

[34] Marsden J E and Hughes J R 1983 Mathematical Foundations of Elasticity (New York: Prentice Hall) (Reprinted by Dover in 1994)

[35] Yavari A, Marsden J E and Ortiz M 2006 On spatial and material covariant balance laws in elasticity J. Math. Phys. 47042903 (53 pages)

[36] Ashurst W T, Kerstein A R, Kerr R M and Gibson C H 1987 Alignment of vorticity and scalar gradient with strain rate in simulated Navier-Stokes turbulence Phys. Fluids 30 2343-53

[37] Naso A and Pumir A 2005 Scale dependence of the coarse-grained velocity derivative tensor structure in turbulence Phys. Rev. E 72056318

[38] Stuart J T 1991 The Lagrangian picture of fluid motion and its implication for flow structures IMA J. Appl. Math. 46147

[39] Stuart J T 1998 Singularities in three-dimensional compressible Euler flows with vorticity Theor. Comput. Fluid Dyn. 10 385-91 